\title{
BIOSURFACE PROCESSING WITH ROLE IN IMPROVING THE OSSEOINTEGRATION OF THE ORAL IMPLANT
}

\author{
Vlad Gabriel Vasilescu ${ }^{1 a}(\mathbb{D})$, Elisabeta Vasilescu ${ }^{2 b^{*}}(\mathbb{D})$, Valentin Sîrbu ${ }^{3 c}(\mathbb{D})$, Lucian Toma Ciocan ${ }^{1 d}(\mathbb{D})$ \\ 'Department of Prosthesis Technology and Dental Materials, Faculty of Dental Medicine, "Carol Davila"University of Medicine and Pharmacy Bucharest, \\ Bucharest, Romania \\ 2Department of Materials and Environment, Faculty of Engineering, "Dunărea de Jos" University of Galați, Galați, Romania \\ ${ }^{3}$ Implant-Prosthetic Therapy Department, Faculty of Dental Medicine, "Carol Davila"University of Medicine and Pharmacy Bucharest, Bucharest, Romania
}

aDDS, PhD, Assistant Professor; e-mail: vlad.vasilescu@umfcd.ro; 0RCIDiD: https://orcid.org/0000-0001-6251-833X

bEng, PhD, Professor; e-mail: elisabeta.vasilescu@ugal.ro; ORCIDiD: https://orcid.org/0000-0002-6794-7368

'DDS, PhD, Assistant Professor; e-mail: dr.sirbu@yahoo.com; ORCIDiD: https://orcid.org/0000-0002-6267-2406

dDDS, PhD, Associate Professor; e-mail: lucian.ciocan@umfcd.ro; ORCIDiD: https://orcid.org/0000-0002-0329-5102

Introduction The osseointegration of the oral implant involves a close contact between the bone and the implant, an important feature that allows the optimal transfer of stresses from the implant to the bone. Achieving and maintaining tissue integration is ensured by a biosurface design with a role in reducing the effect of shear forces on the interface, which can stimulate osteogenesis and facilitate tissue remodelling. Numerous specialized studies describe the method of modifying the biosurface area generated by a certain topography, among them are those that attest to the role of roughness in increasing the number of cells that will adhere to a biosurface with a larger area.

Methodology Research was performed in order to establish the influence of mechanical processing on the micro-roughness of the surface of the samples from the experimental bioalloy Ti10Zr. Atomic force microscopy (AFM), scanning electron microscopy (SEM) and fluorescence microscopy were used to evaluate the experimental results.

Results The study presents the results on the micro-roughness profile and the values of the parameters that characterize the micro-roughness profile groups (2D and 3D) of the biosurfaces processed by grinding and very fine polishing. Aspects regarding the modification of biosurface morphology, compared to mechanical processing, acid corrosion and anodic oxidation, as well as the results regarding cellular behaviour (e.g. adhesion of osteoblasts) to experimentally processed biosurfaces are also presented.

Conclusions The results of this experimental study together with those previously presented in "Controlled Changing of Implantable Bioinert Materials Biosurface "scientific paper compile a synthesis of information on the ability to modify the microtopography of the biosurface of the Ti10Zr alloy, by different ways in order to improve implant osseointegration.

\section{KEYWORDS}

Bioalloy; Experimentally Processed Surface; Micro-roughness; Atomic Force Microscopy; Cell Adhesion.

\section{INTRODUCTION}

The direct and lasting connection between living and reshaped bone that defines the osseointegration of the implant $[1,2,3]$ is determined by the tissue compatibility that influences the healing process of recovery and remodelling, immediately after implantation. Bone healing in the post-implantation period involves a series of cellular and extracellular biological processes at the bone-implant interface, completed with the formation of new bone $[4,5,6]$. The first reactions result in the formation of a clot interface, the biological processes being further controlled by growth and differentiation

Received: January 21, 2021; Revised: February 12, 2021; Accepted: February 15, 2021; Published: February 17, 2021

*Corresponding author: Prof. Dr. Eng. Elisabeta Vasilescu, Department of Materials and Environment, Faculty of Engineering, "Dunărea de Jos"

University of Galați, Str. Domnească, nr.111, Galați, RO-800201 Romania

Tel: 0040236130 208; Fax: 0040236314 463; e-mail: elisabeta.vasilescu@ugal.ro, elisabeta.vasilescu@yahoo.com

Copyright: $\odot 2021$ the Editorial Council for the Stomatology Edu Journal. 

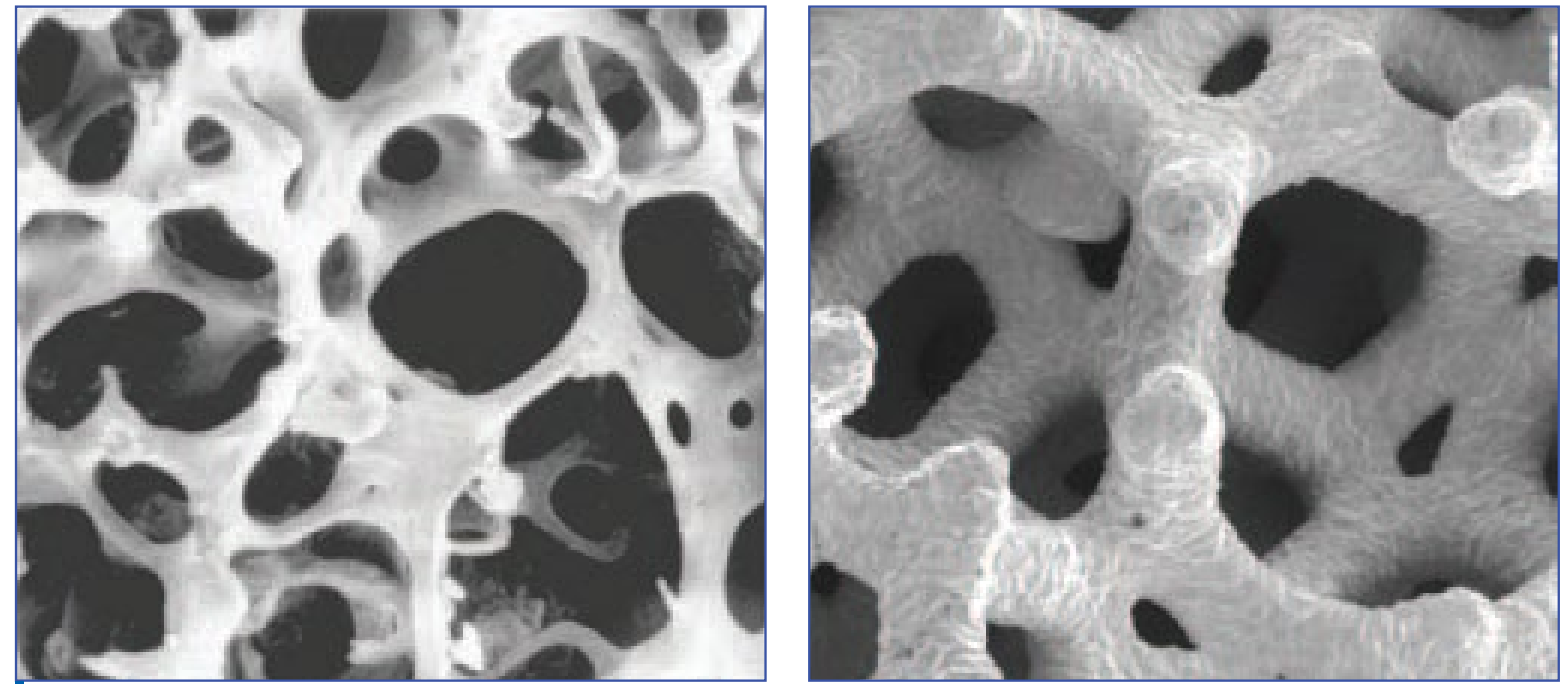

Figure 1. SEM view of trabecular bone (left) and Trabecular Metal Material (right) [18]

factors released by blood cells. They undergo morphological and biochemical changes as an effect of contact with the biosurface $[7,8,9]$. Achieving implant osseointegration, but also maintaining tissue integration are ensured by the presence of a high biocompatible material [10] and a design that stimulates osteogenesis and helps reduce the effect of shear forces on the interface, such as surface roughness and shape characteristics [11,12]. The role of implant surface roughness in stimulating and improving bone growth to implant surface in low bone density structures is highlighted in many specialized studies, which indicated higher post-load failure rates for implants with relatively smooth surfaces compared to implants with low bone density rough surface [13]; Also relevant are the results of comparative studies on the roughness of biosurfaces, obtained by different processing methods, those oxidized with increased roughness have shown a shorter post-implantation healing period due to improved cellular interaction at the bone-implant interface [14]. In summary, the conclusions of the studies on the effect of microrough biosurfaces on the osseointegration process clearly show the indication for the use of titanium "microrough" implants obtained by titanium plasma spraying, or by techniques such as $\mathrm{Al}_{2} \mathrm{O}_{3}$ particle blasting, $\mathrm{TiO}_{2}$ blasting and acid etching. The effects of microrough surfaces, such as faster integration, a larger bone-to-implant contact area compared to titanium implants with a polished or machined surface, have been confirmed by in vitro cell response studies, demonstrating that osteoblasts are sensitive to changes in the roughness of biosurfaces. The clinical benefits mentioned by the authors in recent clinical situations are related to the shortening of the healing period for these implants to 6-8 weeks instead of 12 weeks. The treatment of the implant surface by mechanical, physical or chemical methods but also the differentiated treatment led to a faster healing and a better stability of the implant $[15,16,17]$; It has been shown, especially in low-density bone structures, that implant stability is influenced by implant design and that a combination of microscopic and macroscopic surface topography modification techniques can create a stable boneimplant interface.

In the case of titanium alloys, research on the application of techniques to improve cell interaction and cell development at the interface by intensifying protein adsorption processes has been carried out in order to determine whether bone apposition could be enhanced by a microrough surface obtained by processing techniques such as blasting, acid attack, or combinations thereof. In vitro research of the titanium implant with different surface microtopographies has shown the differentiation of bone and mineralization cells, dependent on roughness. Rough surfaces favour osteointegration of the implant through the attachment and subsequent proliferation of osteoblasts and the size of the implant-bone contact area; Notable results regarding the modification of the biosurface parameters of the titanium implant and titanium alloys were obtained by surface treatment with hydroxyapatite (HA), achieved by various physical, chemical, electrochemical methods such as: Plasma spray, Pulsed Laser Deposition, Chemical Vapor Deposition, Physical Vapor Deposition, HA Blast Coating, etc., noting that regardless of the process applied, metal surfaces are prepared by sandblasting, abrasion or chemical corrosion.

The diversification of the biosurface processing methods but also the advances registered in the design of the oral implant resulted in implants with differentially processed surfaces and well adapted clinically by the positioning mode and the placement area; The implant can have a portion obtained by mechanical surface finishing operations (e.g. by grinding), an engraved portion and a sandblasted portion at high temperatures with an ideal roughness of osseointegration, which ensures the 


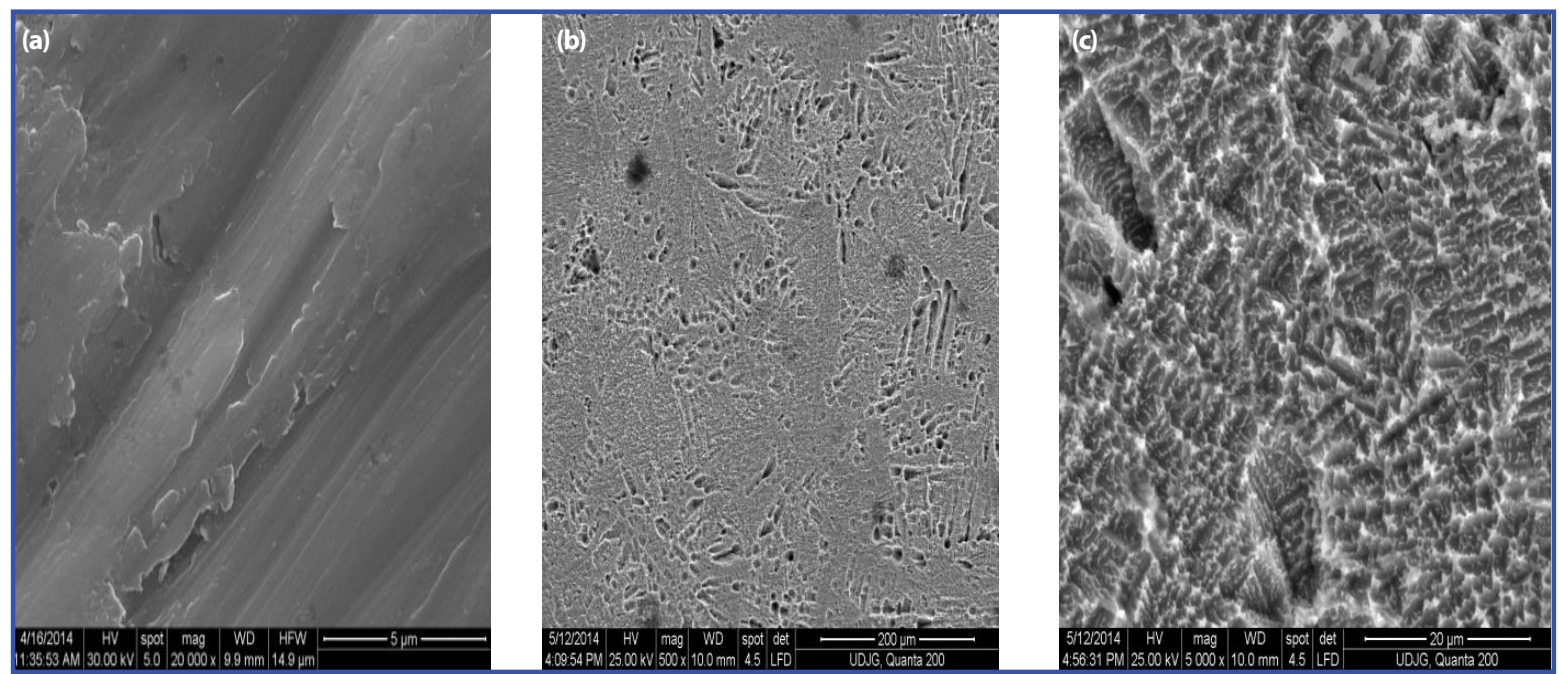

Figure 2. Microscopic aspects of the surface of the Ti10Zr samples experimentally processed through: casting + machining + grinding (a), acid corrosion (b), anodic oxidation (c).

optimal anchoring of the implant. Also, the control of the properties of the tissue-implant interface is well possible in the case of the titanium implant obtained bysintering (LST-LaserSinteredTitanium, 2009) which has a rough surface with micropores of 2-200 $\mu \mathrm{m}$ and a prescribed geometry from the design stage. The healing process begins with the insertion of the implant as this surface stimulates and accelerates the healing of bone tissue, which makes it possible to immediately load the implant into bone of density I, II, III, in much safer conditions. Research in recent years in interdisciplinary fields such as engineering and medicine has led to remarkable results for obtaining biomaterials with high biocompatibility and the promotion of advanced surface processing technologies. Some of these resulted in a porous biomaterial made of TiZrTa alloy with structure and elasticity close to those of bone.

The modern design introduced by the "Trabecular Metal" implant (Fig.1) conceptually revolutionized the theory of osseointegration and introduced the notion of osseoincorporation (growth of bone tissue including in the structure of the implant). Considered the newest discovery in the field of dental implantology, it is the only implant with three-dimensional structure (3D) that mimics bone cell architecture ( $80 \%$ porosity) and systematic nanotextured topography of superficial areas. The trabecular structure of the implant causes the bone to form inside it, resulting in a common body between the implant and the human bone. The implants have a treated surface of SLA type (Sandblasting with Large grit followed by Acid etching), chemically modified and moderately rough which increases the bone-implant contact surface ensuring a period of osseointegration twice less than other implant systems [18].

The paper presents the experimental research conducted in order to study the influence of changes in the microtopography of biosurfaces processed by casting + mechanical processing and casting + mechanical processing + polishing / polishing to mirror gloss, of some samples from the experimental bioalloy TI10Zr.

The research represents the continuation of the study and the completion of the information previously presented in the works "Controlled Changing of Implantable Bioinert Materials Biosurface" [19] and "In Vitro Testing of Materials Biocompatibility with Controlled Chemical Composition" [20], in order to establish the optimal and efficient way to modify biosurface area, and in this way of improving the osseointegration of the oral implant from Ti10Zr, between the mechanical, chemical (acid attack) and electrochemical (anodic oxidation) processing processes.

\section{METHODOLOGY}

In the experiments, samples from the experimental bioalloy with titanium base were used (Ti10Zr/Patent no. 132079/2019). The samples were taken from the molded semi-finished product subsequently subjected to mechanical processing (casting process + mechanical processing by grinding and casting + mirror gloss polishing).

The analysis of the sample surface was performed by scanning electron microscopy (SEM), atomic force microscopy (AFM / EasyScan2 Model), and the investigation and evaluation of the interactions at the interface was performed by in vitro analysis by exposing G292 osteoblasts to these surfaces, under the same conditions with samples from the same bioalloy but treated on the surface by acid attack and anodic oxidation (previously published results) $[19,20]$.

\section{RESULTS}

The results of the scanning electron microscopy analysis (Fig. 2) highlighted the changes in the morphology of the experimental biosurface modified by 


\section{A. Samples taken from cast semi-finished products and subsequently subjected to mechanical processing} (grinding).

a.1.2D images
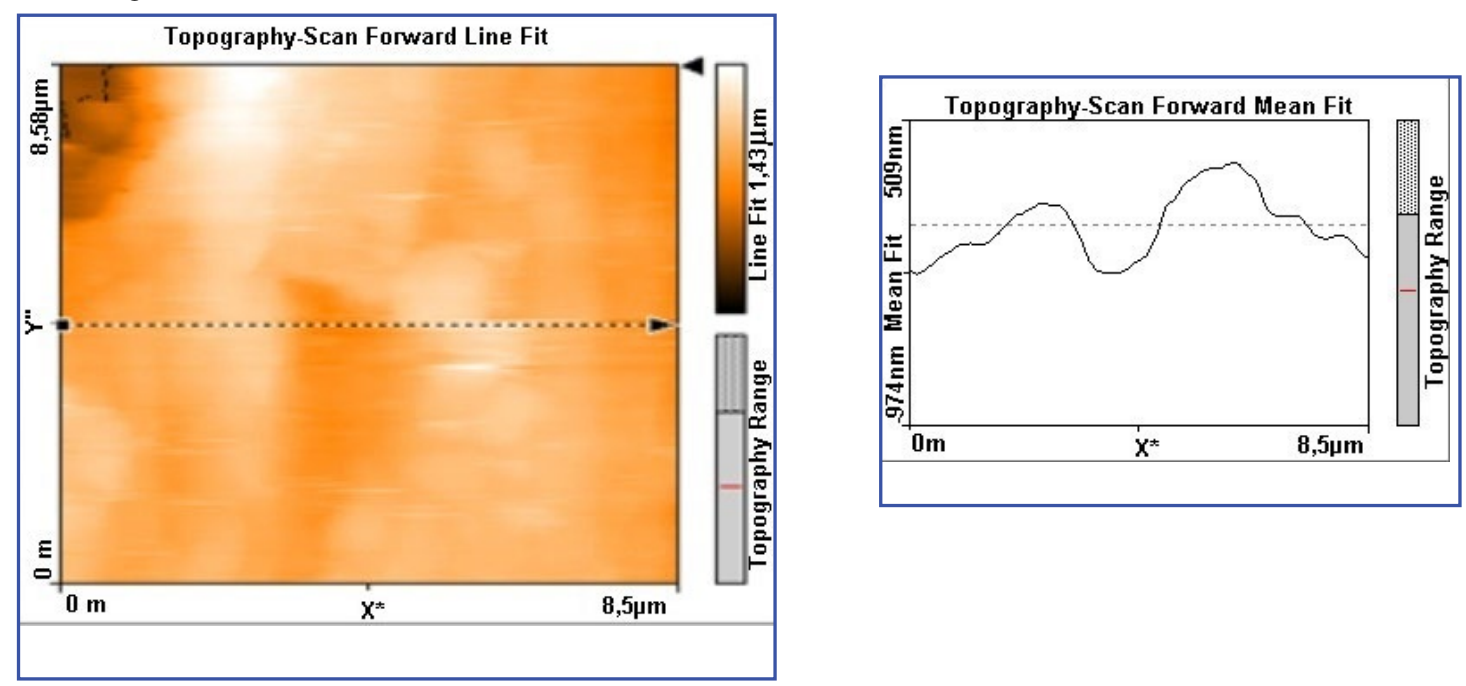

a.2.3D images
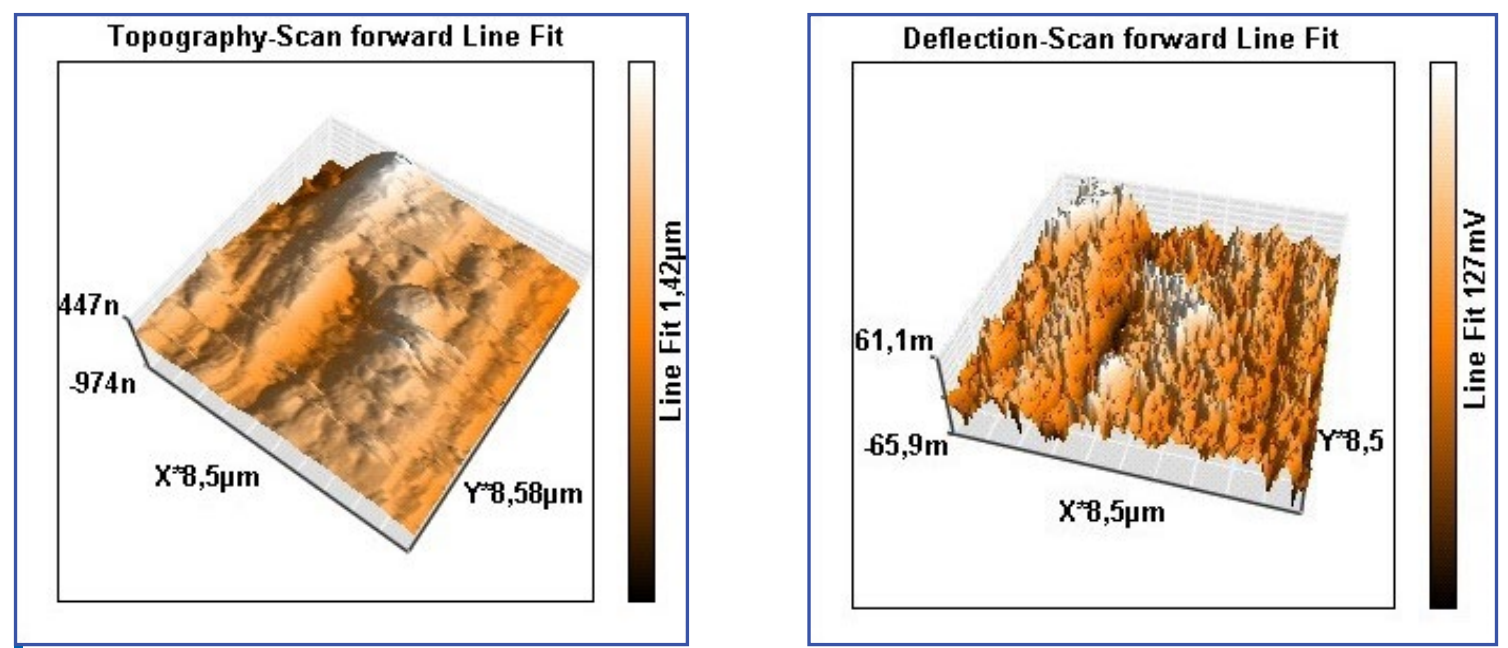

Figure 3. Parameters that characterize the roughness profile.

$R_{a}=125,62 n m, R_{q}=147,75 n m, R y=525,97 n m, R_{p}=266,18 n m, R_{v}=-259,79 n m, R_{m}=-3,628 f m$.

mechanical processing, compared to those obtained by corrosion (acid attack) and anodic oxidation, and the results of atomic force microscopy analysis (Fig. 3 and Fig. 4) illustrates the roughness profile (2D and $3 \mathrm{D}$ images) and shows the measured values of the parameters that characterize the roughness profile groups by mechanical sample processing. Figure 5 shows the aspects regarding the in vitro evaluation of the adhesion of osteoblasts on the surface of the investigated samples.

\section{Remarks:}

The anodic oxidation method allows the development of an oxide layer on the surface of the material with a role in improving the adhesion and fixation properties.

The Ti10Zr alloy samples thus processed provide a special surface configuration, as shown in electron scanning microscopy images (Fig. 2c). The oxide film is a basis for the formation of the osteoinductive matrix. Micro-topographic analysis of the sample surface through atomic force microscopy analysis (AFM) provided useful information on the roughness profile and the values of the parameters that characterize the roughness profile groups. The roughness corresponds in value to surfaces with very fine processing. There are isolated peaks of high roughness, with a rounded shape, characteristic of the surface obtained from mechanical processing (Fig. 4).

The investigation and evaluation of the response of experimentally processed biosurfaces to in vitro cellular behaviour were carried out by exposing G292 osteoblasts to Ti10Zr samples, with surface morphological characteristics conferred by mechanical processing described above, under the same conditions as samples processed by casting, acid corrosion and anodizing.

G292 osteoblasts were seeded in 6-well plates at a density of $5 \times 104$ cells $/ \mathrm{cm}^{2}$ in the presence of Ti10Zr samples with differently processed surfaces; At the same time, cells were cultured in vessels but in 
B. Samples subjected to mechanical processing (grinding + polishing to mirror gloss).

b.1.2D images
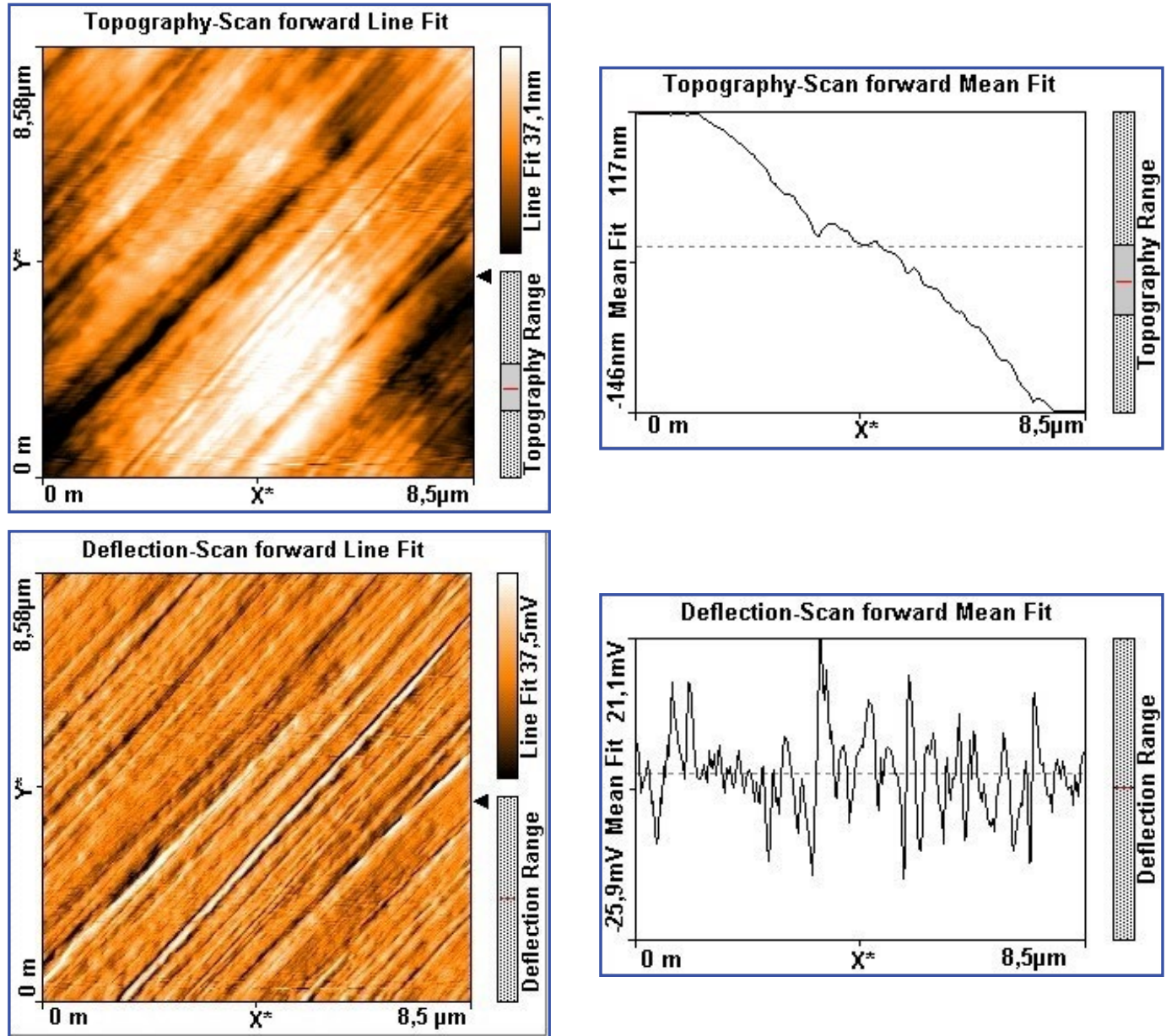

b.2.3D images
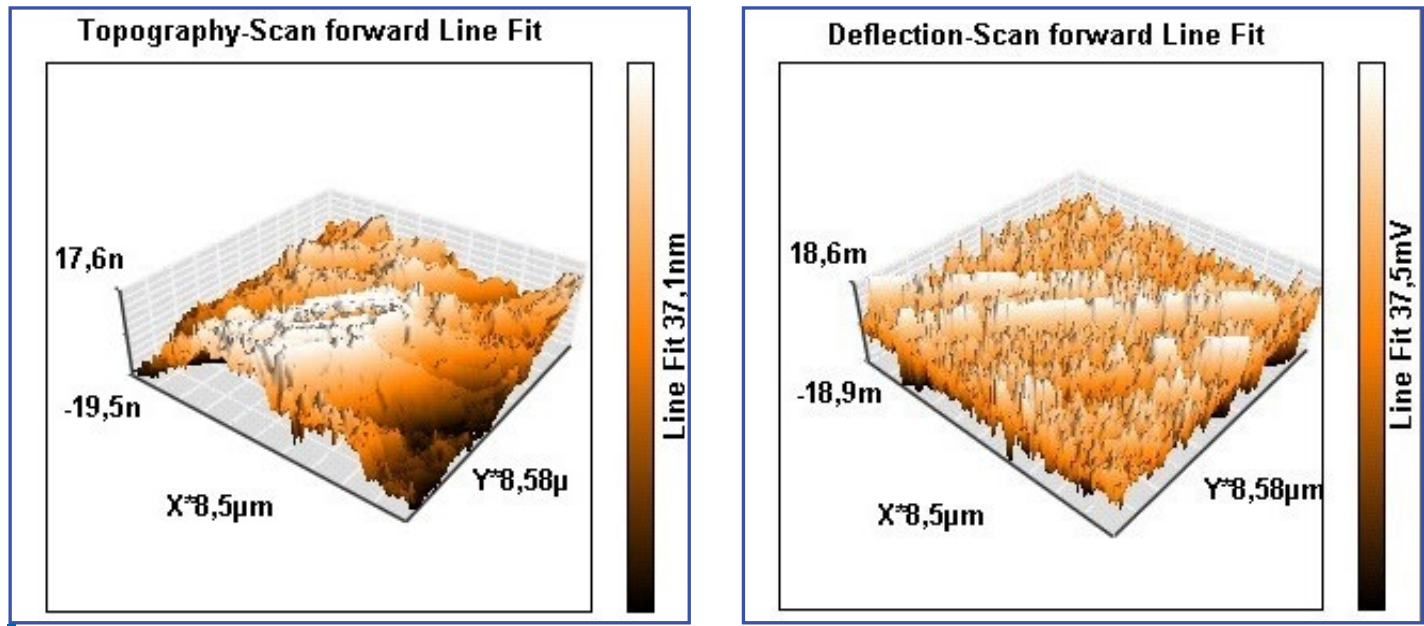

Figure 4. Parameters that characterize the roughness profile.

$R_{a}=7,9282 n m, R_{q}=9,1452 n m, R_{y}=43,537 n m, R_{p}=16,564 n m, R_{v}=-26,973 n m, R^{m}=-3,4725 f m$.

the absence of any material (control). At 24 and 48 hours after incubation, medium was harvested from each well and fluorescent labelling of cytoskeletal actin filaments and intracellular glutathione was performed (Fig. 5).

Examination by fluorescence microscopy of the architecture of actin filaments revealed that the cells grew in a single layer, showed an osteoblast - like phenotype and there were no differences from the control. The experimental results demonstrate, for all ways of processing the biosurface of Ti10Zr samples (acid attack, anodizing, mechanical processing), a good adhesion of osteoblasts, especially to oxidized ones, which have cells with a well-organized actin cytoskeleton, interconnected and with cell densities comparable to those of the control (in the absence of sample material). 


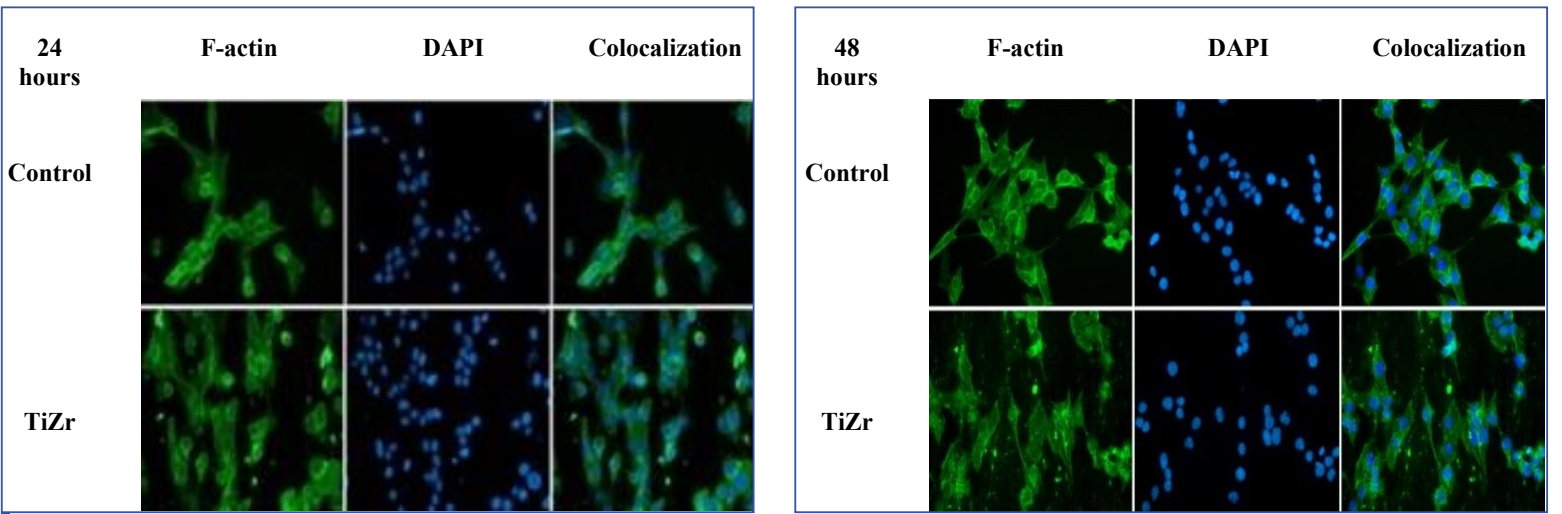

Figure 5. Highlighting the actin cytoskeleton by fluorescent labelling of F-actin with phalloidin-FITC (DAPI nucleus counter colouring) in osteoblasts grown for 24 and 48 hours on the surface of the culture vessel/Control and Ti10Zr alloy [21].

\section{DISCUSSIONS}

The study of the influence of the mechanical processing method on the micro-roughness profile and the values of the micro-roughness parameters determines the cellular behaviour, tested by evaluating the adhesion and the distribution of osteoblasts on surfaces thus processed.

In vitro testing of cellular behaviour, which provides the information needed to understand the mechanism by which surface micro-roughness controls the cellular response, proves that different changes in topography lead to differentiated responses, at least in cell distribution mode.

Corroborating the results obtained in the mechanical processing of the surface with those obtained in the chemical processing (corrosion or anodic oxidation) of the Ti10Zr bioalloy, differences were observed both in terms of the micro-roughness profile and the values of the micro-roughness parameters, but also in terms of the mode of cell spread on these surfaces. Mechanically machined surfaces contribute to the obtaining of microrough biosurfaces. The values characterizing the parameters of the microroughness profile are not the same for differently machined surfaces (with different micro-roughness profile) and are significantly lower for surfaces with fine machining (samples with almost smooth surface, polished).

Compared to the surfaces processed by corrosion or anodic oxidation, those obtained by mechanical processing (regardless of the processing method) in addition to a good adhesion of osteoblasts, presented an orientation of adherent cells depending on the direction of mechanical processing of samples. It has been observed that any further processing plays a decisive role in how the cells are oriented and adhered to these surfaces and annihilate the influence of previous processing.

\section{CONCLUSIONS}

The research highlights the possibilities of processing the biosurface of the implant with a role in improving the biological processes at the implant-tissue interface in the immediate post-implantation period. The results of this experimental study together with those previously presented in "Controlled Changing of Implantable Bioinert Materials Biosurface" provides useful information on the ability of the Ti10Zr bioalloy to modify its microtopography of the biosurface, by different ways to improve implant osseointegration. The results of the research that aimed to establish the optimal way to modify the biosurface area through more ways of processing are presented. The modification of the biosurface parameters (microroughness) was evaluated, as well as their influence on the adhesion of osteoblasts and the cell proliferation capacity on the experimentally processed surfaces.

The conclusions of the research are that the increase of the biosurface area by modifying the morphology and/or micro-roughness either by corrosion, by oxidation or coarse either high-precision mechanical processing, has been demonstrated and denotes the ability of Ti10Zr bioalloy to improve biosurface characteristics. However, some observations of the experimental study should be noted, which may be of interest in selecting one or another of the experienced processing methods, as follows:

a. considering any of the processes applied in coatings to increase the bioactivity of the metal surface, they require prior preparation by blasting, abrasion or chemical corrosion.

b. the increase of the biosurface area by creating rough or microporous surfaces facilitates the cell adhesion processes and the growth of the trabecular bone directly on the surface of the titanium implant, shortening the post-implantation healing period.

c. the anodic oxidation method creates surfaces covered with a uniform, continuous, adherent oxide film with a special morphological configuration with a decisive role in stimulating the processes at the interface.

d. mechanically processed surfaces contribute to obtaining biosurfaces with a profile of microroughness and values of roughness parameters depending on the degree of processing and 
influence the orientation of the adhered cells according to the direction of processing.

e. however, it was not noticed for corroded or anodized Ti10Zr, suggesting that additional processes have changed the properties of these surfaces, and thus have decisively influenced cell orientation and adherence;

f. these differences detected in surfaces with different processing could have a major influence on how osteoblasts managed to adapt and be the best option for dental implant.

\section{CONFLICT OF INTEREST}

The authors declare no conflict of interest.

\section{ACKNOWLEDGMENTS}

None.

\section{REFERENCES}

1. Brånemark PI. Osseointegration and its experimental background. J Prosthet Dent. 1983;50(3):399-410. doi: 10.1016/ s0022-3913(83)80101-2. PMID: 6352924.

Full text links CrossRef PubMed Google Scholar WoS 2. Brånemark PI, Zarb GA, Albrektsson T. Tissue-integrated prostheses: osseointegration in clinical dentistry. 1st edition. Chicago, IL: Quintessence Pub Co; 1985. ISBN-13: 9780867151299.

3. Jokstad A. Osseointegration and dental implants. John Wiley \& Sons; 2008. ISBN: 978-0-813-81341-7, Available from: Osseointegration and Dental Implants |Wiley Online Books 4. Albrektsson T. Bone tissue response (p. 129-143) in: Brånemark PI, Zarb GA, Albrektsson T. Tissue-integrated prostheses: osseointegration in clinical dentistry. 1st edition. Chicago, IL: Quintessence Pub Co; 1985. ISBN-13: 978-0867151299 5. Mavrogenis AF, Dimitriou R, Parvizi J, Babis GC. Biology of implant osseointegration. J Musculoskelet Neuronal Interact. 2009;9(2):61-71. PMID: 19516081.

Full text links PubMed Google Scholar

6. Vidyasagar L, Apse P. Dental implant design and biological effects on bone-implant interface. Stomatologija. 2004;6(2):5154. Available from: https://sbdmj.com/042/042-04.pdf. [cited 2021, Feb 10].

Google Scholar

7. Ramazanoglu M, Oshida Yoshiki. Osseointegration and bioscience of implant surfaces - current concepts at bone-implant interface, August 29th 2011. doi: 10.5772/16936. Available from: https://www.intechopen.com/books/implant-dentistry-a-rapidlyevolving-practice/osseointegration-and-bioscience-of-implantsurfaces-current-concepts-at-bone-implant-interface Google Scholar

8. Anil S, Anand PS, Aghamdi H, Jansen JA. Dental implant surface enhancement and osseointegration, August 29th 2011 doi:10.5772/16475. Available from: https://www.intechopen. com/books/implant-dentistry-a-rapidly-evolving-practice/ dental-implant-surface-enhancement-and-osseointegration Google Scholar

9. Kohn DH. Overview of factors important in implant design. $J$ Oral Implantol. 1992;18(3):204-219. PMID: 1289556.

PubMed Google Scholar Scopus

10. Frenkel SR, Simon J, Alexander $\mathrm{H}$, et al. Osseointegration on metallic implant surfaces: effects of microgeometry and growth factor treatment. J Biomed Mater Res. 2002;63(6):706-713. doi: 10.1002/jbm.10408. PMID: 12418014.

CrossRef PubMed Google Scholar Scopus WoS

11. O'Brien WJ. Dental materials and their selection. 4th edition. 4350 Chandler Dr Hanover Park, IL 60133: Quintessence Publishing Co, Inc; 2009. ISBN: 978-0867154375.

Google Scholar

\section{AUTHOR CONTRIBUTIONS}

VGV: participated in the elaboration, writing and translation of the paper and contributed to the introductory part (synthesis of specialized information on the topic), to establishing the experimental conditions, interpreting the results and formulating the research conclusions. EV: contributed as follows: characterization of the materials researched and the interpretation of the results obtained in the investigation by advanced methods (SEM Microscopy, EDS Analysis). VS: participated in the writing and translation of the paper and contributed to structuring the bibliographical references. LTC: participated in the research of the documentary sources (bibliographical references), in the structuring of the research conditions and methodology and in the elaboration of the abstract.
12. Nasatzky E, Gultchin J, Schwartz Z. [The role of surface roughness in promoting osteointegration]. Refuat Hapeh Vehashinayim (1993). 2003;20(3):8-19, 98. Hebrew. PMID: 14515625.

PubMed Google Scholar

13. Cochran DL. A comparison of endosseous dental implant surfaces. J Periodontol. 1999;70(12):1523-1539. doi: 10.1902/ jop.1999.70.12.1523. PMID: 10632528.

Full text links CrossRef PubMed Google Scholar WoS

14. Rasmusson L, Kahnberg KE, Tan A. Effects of implant design and surface on bone regeneration and implant stability: an experimental study in the dog mandible. Clin Implant Dent Relat Res. 2001;3(1):2-8. doi: 10.1111/j.1708-8208.2001.tb00123.x. PMID: 11441539.

Full text links CrossRef PubMed Google Scholar Scopus

15. Maniatopoulos C, Pilliar RM, Smith DC. Threaded versus porous-surfaced designs for implant stabilization in boneendodontic implant model. J Biomed Mater Res. 1986;20(9):13091333. doi: 10.1002/jbm.820200907. PMID: 3782184.

CrossRef PubMed Google Scholar Scopus WoS

16. Abraham CM. A brief historical perspective on dental implants, their surface coatings and treatments. Open Dent J. 2014 May 16;8:50-55. doi: 10.2174/1874210601408010050. PMID: 24894638; PMCID: PMC4040928.

Free PMC Article CrossRef PubMed Google Scholar Scopus

17. Le Guéhennec L, Soueidan A, Layrolle P, Amouriq Y. Surface treatments of titanium dental implants for rapid osseointegration. Dent Mater. 2007;23(7):844-854. doi: 10.1016/j. dental.2006.06.025. PMID: 16904738.

Full text links CrossRef PubMed Google Scholar Scopus WoS 18. Collins M, Bassett J, Wen HB, et al. Zimmer ${ }^{\circledR}$ trabecular metal $^{\mathrm{TM}}$ dental implant research: a brief overview. Zimmer Clinical Paper. 2012;V(9)

Google Scholar

19. Vasilescu E, Vasilescu VG, Pătrașcu I, Frățilă C. Controlled changing of implantable bioinert materials biosurface. Rev Rom Mater. 2016;46(1):11-16. Available: http://solacolu.chim.upb.ro/ p11-16w.pdf Google Scholar WoS

20. Vasilescu VG, Stan MS, Pătrașcu I, et al. In vitro testing of materials biocompatibility with controled chemical composition. Rev Rom Mater. 2015;45(4):315-323. Available: http://solacolu. chim.upb.ro/pg315-323w.pdf Google Scholar WoS 


\section{Vlad Gabriel VASILESCU \\ DDS, PhD, Assistant Professor \\ Department of Prosthesis Technology and Dental Materials Faculty of Dental Medicine “Carol Davila” University of Medicine and Pharmacy Bucharest Bucharest, Romania}

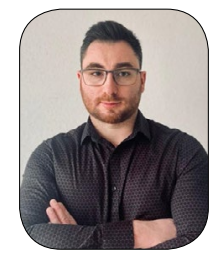

Dr. Vlad Gabriel Vasilescu is Assistant Professor at the Department of Prosthesis Technology and Dental Materials, Faculty of Dental Medicine of "Carol Davila" University of Medicine and Pharmacy Bucharest. In 2016, he obtained the PhD in Dentistry with the thesis entitled "Contributions to the study of biocompatible metal materials for oral implantology". Areas of interest in the research activity: obtaining and characterizing dental materials and highly biocompatible materials, characterizing implantable systems in relation to the biocompatibility of materials and surface microtopography. Disseminating research results involves communications at prestigious scientific events and publications in specialized journals, awards and distinctions: Gold Medal for "High biocompatibility alloy for dental implants", Diploma of Excellence for the works"Electron Microscopy Studies of Depositing Metallic Silver with Antibacterial Role on the TiZr Dental Implant Surface".

\section{Ouestions}

\section{Improving the osseointegration of the implant is possible by:}

$\square$ a. The modification of the microtopography that determines the increase of the biosurface area;

b. Use of implants with smooth (unprocessed) surfaces;

ac. Early implant loading;

$\square$ d. The use of materials with high fatigue resistance.

\section{Changes in biosurface morphology by anodic oxidation have the following effect:}

$\square$ a. Increase of the oxide layer on the implant surface;

ab. Improve osteoinductive properties;

c. Decrease biological processes at the tissue-implant interface;

$\square$ d. Decreased cell adhesion.

\section{Maintaining tissue integration is improved:}

$\square$ a. By an implant biosurface design that increases the effect of shear forces;

ab. By factors that diminish the primary bone-implant stability;

ac. By a design of the implant biosurface that reduces the effect of shear forces;

$\square$ d. In structures with low bone density.

\section{The role of the microroughness of the implant surface is:}

a. To inhibit the growth of bone tissue to the surface of the implant;

ab. To improve protein adsorption in the cellular interaction at the interface between tissue and biomaterial;

c. To reduce the bone-implant contact surface;

$\square$ d. To prevent the interfacial reaction in the post-implantation period. 